\title{
Surface Based Atlas Matching of the Brain Using Deformable Surfaces and Volumetric Finite Elements
}

\author{
Matthieu Ferrant ${ }^{1}$, Olivier Cuisenaire ${ }^{2}$, Benoît Macq, Jean-Philippe Thiran ${ }^{2}$, \\ Martha E. Shenton ${ }^{3}$, Ron Kikinis ${ }^{3}$, and Simon K. Warfield ${ }^{3}$ \\ ${ }^{1}$ Université catholique de Louvain (UCL/TELE), B-1348 Louvain-la-Neuve, Belgium. \\ 2 Swiss Federal Institute of Technology (EPFL/LTS), CH-1015 Lausanne, Switzerland. \\ 3 Surgical Planning Laboratory, Brigham and Women's Hospital, Boston, MA02115 USA.
}

\section{Introduction}

The automatic identification and localization of structures in magnetic resonance (MR) brain images are a major part of the processing work for the neuroradiologist in numerous clinical applications, such as functional mapping and surgical planning. To aid in this task, a considerable amount of research has been directed toward the development of 3D standardized atlases of the human brain (e.g. [5]). These provide an invariant reference system and the possibility of template matching, allowing anatomical and functional structures in new scans to be identified and analyzed.

There are mainly two types of methods for doing deformable atlas matching : surface-based and volume based methods. Surface based methods deform key surfaces of the atlas onto the target image and interpolate the surface displacement to obtain a fully volumetric mapping (e.g. [3]). Volumetric methods compute a deformation field that minimizes a similarity criterion between the atlas and the target image under a given regularization constraint (e.g. elastic). To reach convergence, the computations must be done in a multi-resolution fashion (e.g. [1]). The main issue with both methods is the initialization of the volume or surfaces.

In this paper, we propose a surface based method with automatic initialization of the deformable surfaces using a global parametric transformation. We use an elastic volumetric finite element (FE) deformation model to infer a volumetric deformation field from the obtained surface deformations. The method provides us with a fully automated volumetric mapping of the atlas onto a target image.

\section{Atlas Matching Algorithm}

The landmark deformable surfaces of the atlas (cortical surface and ventricles) are initialized on the target image using a 2 nd degree polynomial transform that minimizes a distance measure of the landmark surfaces to the distance transform of the corresponding objects in the target image [2]. The objects are segmented out of the target image using a directional watershed algorithm [2]. We then match the initialized landmark surfaces onto the target image using our deformable surface matching algorithm [4], as illustrated in Figure 1

We infer a volumetric deformation field from the surface deformations by applying the surface displacements obtained after global registration and active surface deformation as a boundary condition to a volumetric FE tetrahedral mesh we have extracted from the atlas image [4]. After the atlas's volumetric FE mesh has been deformed by the boundary surfaces, the obtained deformation field can be interpolated back onto the image grid to produce a volumetric mapping from the atlas space onto the target image space [4]. Figure 2] illustrates the different volumetric deformation stages of atlas brain onto a target brain. The volumetric mapping can then be used to outline other objects in the target image. 

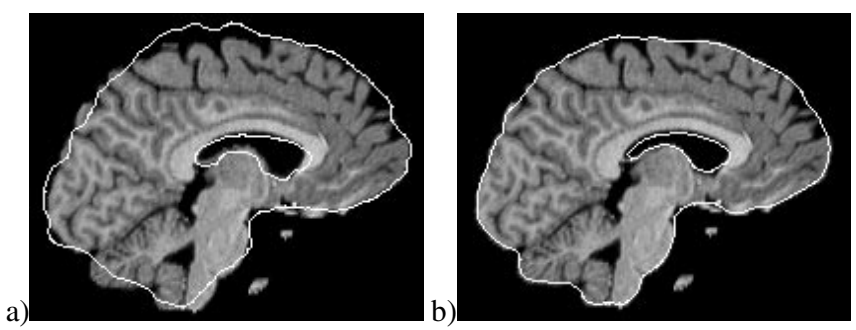

Fig. 1. a) cut through deformable surfaces after 2nd degree polynomial transform overlayed on corresponding cuts through target image. b) Same cut but after active surface deformation.
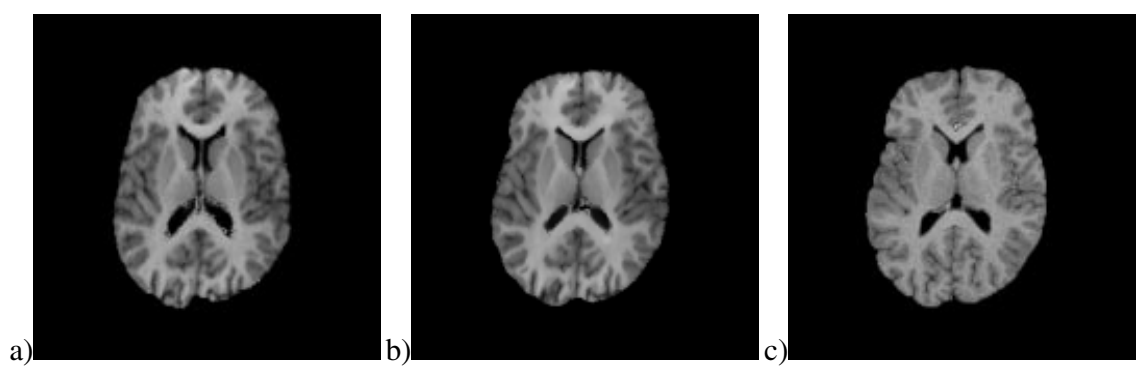

Fig. 2. Volumetric deformations of the atlas MR image illustrating the transformation steps of the algorithm. a) Slice of deformed volume after 2 nd degree registration of ventricles and brain surface. b) Same slice after active surface deformation. c) Same slice of target image.

\section{Conclusion}

We have presented a surface based deformable atlas matching algorithm that automatically initializes the deformable surfaces. An arbitrary number of surfaces can be matched to drive the volumetric warp of the atlas onto a target image, and the finite element formulation of the volumetric match allows for variable elastic deformations of the atlas's objects. Our preliminary experiments show very good correlation of the deformed atlas with the target image.

\section{References}

1. G.E. Christensen, S.C. Joshi, and M.I. Miller. Volumetric Transformation of Brain Anatomy. IEEE Trans. Med. Imag., 16(6):864-877, December 1997.

2. O. Cuisenaire. Distance Transformations : Fast Algorithms and Applications to Medical Image Processing. $\mathrm{PhD}$ thesis, Telecommunications Laboratory, Université catholique de Louvain, B-1348 Belgium, 1999.

3. C. Davatzikos. Spatial Transformation and Registration of Brain Images Using Elastically Deformable Models. Computer Vision and Image Understanding, 66(2):207-222, May 1997.

4. M. Ferrant, S.K. Warfield, A. Nabavi, B. Macq, F. Jolesz, and R. Kikinis. Registration of 3D Intraoperative MR Images of the Brain Using a Finite Element Biomechanical Model. In Anthony M. DiGioia and Scott Delp, editors, MICCAI 2000: Third International Conference on Medical Robotics, Imaging And Computer Assisted Surgery; 2000 Oct 11-14; Pittsburgh, USA, pages 19-28. Springer, 2000.

5. R. Kikinis, M.E. Shenton, D.V. Iosifescu, R.W. McCarley, P. Saiviroonporn, H.H. Hokama, A. Robatino, D. Metcalf, C.G. Wible, C.M. Portas, R. Donnino, and F.A. Jolesz. A Digital Brain Atlas for Surgical Planning, Model Driven Segmentation and Teaching. IEEE Trans. on Visualization and Computer Graphics, 2(3):232-241, 1996. 